\title{
Unicystic Ameloblastoma
}

National Cancer Institute

\section{Source}

National Cancer Institute. Unicystic Ameloblastoma. NCI Thesaurus. Code C39756.

An ameloblastoma that presents as a unilocular cyst. It usually arises from the mandible. 